\title{
Bistable dark solitons of a cubic-quintic Helmholtz equation
}

\author{
J. M. Christian and G. S. McDonald \\ Joule Physics Laboratory, School of Computing, Science and Engineering, \\ Materials \& Physics Research Centre, University of Salford, Salford M5 4WT, U.K. \\ P. Chamorro-Posada \\ Departamento de Teoría de la Señal y Comunicaciones e Ingeniería Telemática, \\ Universidad de Valladolid, ETSI Telecomunicación, Campus Miguel Delibes s/n, \\ 47011 Valladolid, Spain
}

Submitted to Physical Review A

\begin{abstract}
We report, to the best of our knowledge, the first exact analytical bistable dark spatial solitons of a nonlinear Helmholtz equation with a cubic-quintic refractive-index model. Our analysis begins with an investigation of the modulational instability characteristics of Helmholtz plane waves. We then derive a dark soliton by mapping the desired asymptotic form onto a uniform background field, and obtain a more general solution by deploying rotational invariance laws in the laboratory frame. The geometry of the new soliton is explored in detail, and a range of new physical predictions is uncovered. Particular attention is paid to the unified phenomena of arbitrary-angle off-axis propagation and non-degenerate bistability. Crucially, the corresponding solution of paraxial theory emerges in a simultaneous multiple limit. We conclude with a set of computer simulations that examine the role of Helmholtz dark solitons as robust attractors.
\end{abstract}

PACS numbers: 05.45.Df, 42.65.Hw, 42.65.Sf 


\section{INTRODUCTION}

Spatial solitons are robust self-localized optical beams that evolve with a stationary intensity profile when diffractive spreading is exactly opposed by medium nonlinearity. Two-dimensional (2D) planar waveguide geometry, where there is a longitudinal direction and a single (effective) transverse direction, permits such beams to be self-stabilizing. This innate stability against perturbations makes $2 \mathrm{D}$ spatial solitons ideal candidates for "building blocks" in future integrated-optic devices and architectures [1].

Angular considerations play a pivotal role in nearly all applications in photonics. Two examples that are fundamental to device modelling are: (i) the multiplexing of two (or more) beams [2], and (ii) the reflection/refraction of beams incident at a material interface [3]. Over the past few decades, these classic configurations have been described within nonlinear Schrödinger-type frameworks. Such paraxial analyses have yielded a great deal of insight into a wealth of potentially-exploitable phenomena, but they are inherently limited to considering near-negligible angles (with respect to the reference direction). The full angular characteristics of multiplexing [4] and interface [5] phenomena have only recently been described by developing formalisms based on the underlying nonlinear Helmholtz equations.

Many contexts for optical-switching [6,7] and optical-memory [8] applications are based upon bistable dynamics, where the system's input-output curve has a characteristic "S" shape. The origin of these hysteretic response curves tends to lie in external boundary conditions, typically cavity feedback. Material interfaces can also give rise to regimes of bistable operation $[9,10]$. 
Some two decades ago, Kaplan introduced a type of bistability that, crucially, does not require external boundary conditions [11]. This intrinsic phenomenon [12] is potentially useful for switching applications exploiting spatial solitons in planar waveguides [13], as opposed to cavity solitons [6-8,14]. For a wide class of nonlinearity, there is often a parameter regime where one finds the coexistence of degenerate bright solitons - that is, beam solutions with different propagation constants but the same power $[15,16]$. While the ubiquitous Kerr nonlinearity is excluded from this category, materials with more involved refractive nonlinearities (e.g., cubic-quintic and saturable) offer greater flexibility for potential device designs. In this paper, we consider a type of bistability, proposed by Gatz and Herrmann [17], that describes non-degenerate solitons: one can find pairs of beam solutions that have the same full-width-at-half-maximum (FWHM) but different powers $[18,19]$. In recent papers [20], we unified the physical contexts of nondegenerate bistability and oblique (off-axis) propagation through proposal and analyses of Helmholtz equations with cubic-quintic and saturable nonlinearities. Exact analytical bright solitons were derived, and parameter regimes were identified where solutions have a bistable characteristic. Solutions lying on both branches were found to behave as robust attractors.

Bistable dark solitons have been known in paraxial wave optics for many years [21-23]. However, dark-soliton phase topology (a localized grey "dip" that modulates a uniform background) means that the notion of degenerate bistability becomes more subtle [24]. However, non-degenerate bistability is still physically meaningful and can be easily interpreted.

In this paper, we are concerned with the angular properties of bistable dark solitons. In Section II, a cubic-quintic Helmholtz model is proposed. Exact analytical 
dark solitons for this new governing equation are then derived by mapping the solution asymptotics directly onto a plane-wave background; both forward- and backward-propagating beams are obtained. A bistable characteristic is investigated and, in an appropriate multiple limit, the classic paraxial soliton [23] can be recovered from the more general Helmholtz solution. In Section III, the stability of the new soliton is examined through computer simulations, and we conclude, in Section IV, with some remarks about the potential application of this work.

\section{HELMHOLTZ SOLITON THEORY}

\section{A. Field and envelope equations}

We consider a transverse-electric (TE) polarized continuous-wave scalar electric field $E(x, z, t)=E(x, z) \exp (-i \omega t)+$ c.c. with angular frequency $\omega$. The spatial part $E(x, z)$ is assumed to vary on a scalelength that is much larger than the free-space optical wavelength $\lambda$, and thus satisfies the Maxwell field equation $[25,26]$,

$$
\left(\frac{\partial^{2}}{\partial z^{2}}+\frac{\partial^{2}}{\partial x^{2}}\right) E(x, z)+\frac{\omega^{2} n^{2}}{c^{2}} E(x, z)=0 .
$$

In uniform media, there is no physical distinction between $x$ and $z$. This spatial symmetry appears in Eq. (1) as invariance under the permutation $x \leftrightarrow z$, and diffraction is thus fully-2D (occurring in both $x$ and $z$ ). Explicit $x-z$ equivalence permits multiple beams to propagate and interact at arbitrary angles (with respect to the reference direction) and orientations (with respect to each other) [4].

The refractive index of the medium is taken to be $n=n_{0}+n_{N L}\left(|E|^{2}\right)$, where $n_{0}$ is the linear index at frequency $\omega$, the intensity-dependent contribution is $n_{N L}\left(|E|^{2}\right)=-$ $n_{2}|E|^{2}+n_{4}|E|^{4}$, and $n_{2}$ and $n_{4}$ are (small, real) coefficients. It is assumed throughout that $n_{2}>0$ (so that the Kerr contribution is always of the defocusing type) but $n_{4}$ may 
be of either sign. For weak optical nonlinearity, one has that $n^{2} \sqcup n_{0}^{2}+2 n_{0} n_{N L}\left(|E|^{2}\right)$. By choosing the $z$ axis as the reference direction and writing $E(x, z)=E_{0} u(x, z) \exp (i k z)$, an equation for the dimensionless envelope $u(x, z)$ can be derived without further approximation:

$$
\kappa \frac{\partial^{2} u}{\partial \zeta^{2}}+i \frac{\partial u}{\partial \zeta}+\frac{1}{2} \frac{\partial^{2} u}{\partial \xi^{2}}-|u|^{2} u+\alpha|u|^{4} u=0
$$

Here, $\zeta=z / L_{D}$ and $\xi=2^{1 / 2} x / w_{0}$, where $L_{D}=k w_{0}^{2} / 2$ is the diffraction length of a reference Gaussian beam. The inverse beam width is quantified by $\kappa=1 /\left(k w_{0}\right)^{2}=$ $\varepsilon^{2} / 4 \pi^{2} n_{0}^{2}<<O(1)$, where $\varepsilon \equiv \lambda / w_{0}, k=n_{0} k_{0}$ and $k_{0}=\omega / c=2 \pi / \lambda$. Finally, $E_{0}=$ $\left(n_{0} / n_{2} k L_{D}\right)^{1 / 2}$ and $\alpha=E_{0}{ }^{2}\left(n_{4} / n_{2}\right)$. The full generality of $\partial_{z z}$ in Eq. (1) has been retained in Eq. (2) by omitting the slowly-varying envelope approximation.

Ultranarrow-beam corrections [27-30] are unimportant in Helmholtz modelling: we assume broad beams throughout (where $w_{0}>>\lambda$ ), so that $\varepsilon<<O(1)$ is always rigorously satisfied. The polarization-scrambling term $\nabla(\nabla \cdot \mathbf{E})$ in Maxwell's equations, which couples the transverse and longitudinal electric field components, can thus be safely neglected so that waves are assigned a purely transverse (scalar) character. Since vector effects are redundant for a broad on-axis beam, they are clearly also redundant for the same beam propagating obliquely to the reference direction. This follows from the fact that on- and off-axis configurations are linked by a rotational transformation, and all physical properties of the beam must be independent of the relative orientation of the observer's coordinate axes. 


\section{B. Plane waves and modulational instability}

It is instructive to consider first the plane waves of Eq. (2). Since the governing equation is bi-directional (due to the retention of $\kappa \partial_{\zeta \zeta}$ in the linear wave operator), one expects to find both forward- and backward-propagating solutions [26]. By substituting $u(\xi, \zeta)=\rho_{0}{ }^{1 / 2} \exp \left[i\left(k_{\xi} \xi+k_{\zeta} \zeta\right)\right]$ into Eq. (2), where $\rho_{0}$ is the intensity, one arrives at the nonlinear dispersion relation $\kappa k_{\zeta}^{2}+k_{\zeta}+1 / 2 k_{\xi}^{2}+\rho_{0}\left(1-\alpha \rho_{0}\right)=0$. The elliptic (i.e., quadratic) nature of this relation allows one to identify two distinct solutions,

$$
k_{\zeta}=-\frac{1}{2 \kappa} \pm \frac{1}{2 \kappa} \sqrt{1-4 \kappa\left[\frac{1}{2} k_{\xi}^{2}+\rho_{0}\left(1-\alpha \rho_{0}\right)\right]},
$$

which correspond to forward $(+)$ and backward $(-)$ fields. By considering the transformation laws of Eq. (2) [26], one can connect $k_{\xi}$ to a conventional transverse velocity parameter $V$ through

$$
k_{\xi} \equiv \mp V \sqrt{\frac{1-4 \kappa \rho_{0}\left(1-\alpha \rho_{0}\right)}{1+2 \kappa V^{2}}} .
$$

In turn, $V$ is related to the propagation angle $\theta$ of the plane wave (in unscaled space, and with respect to the $\pm z$ direction, respectively) through

$$
\tan \theta=\sqrt{2 \kappa} V
$$

(see Fig. 1). By combining Eqs. (3a) and (3b), the two plane wave solutions may be expressed as

$$
\begin{aligned}
u(\xi, \zeta) & =\sqrt{\rho_{0}} \exp \left[ \pm i \sqrt{\frac{1-4 \kappa \rho_{0}\left(1-\alpha \rho_{0}\right)}{1+2 \kappa V^{2}}}\left(-V \xi+\frac{\zeta}{2 \kappa}\right)\right] \\
& \times \exp \left(-i \frac{\zeta}{2 \kappa}\right)
\end{aligned}
$$

where the complex-exponential $\exp (-i \zeta / 2 \kappa) \equiv \exp (-i k z)$ is the rapid-phase term inherent to Helmholtz envelope solutions. Inclusion of $\kappa \partial_{\zeta \zeta}$ thus allows one to 
transform between normalized and laboratory coordinate systems without approximation.

When $\alpha>0$ (competing cubic-quintic nonlinearity), each Helmholtz plane wave becomes modulationally unstable against long-wave perturbations in the band $\left|K_{\xi}\right|<2\left[\rho_{0}\left(-1+2 \alpha \rho_{0}\right)\right]^{1 / 2}$ when $\rho_{0}>1 / 2 \alpha[31]$. When $\alpha<0$ (purely defocusing nonlinearity), there is no modulational instability. Interestingly, $\rho_{0}$ must satisfy $4 \kappa \rho_{0}\left(1-\alpha \rho_{0}\right) \leq 1$, otherwise $k_{\zeta}$ becomes pure imaginary and the wave is evanescent in its propagation direction. The origin of this requirement, which is always met in practice, is that the total refractive index (squared) due to the plane wave, i.e., $n_{0}^{2}+$ $2 n_{0} n_{N L}\left(E_{0}{ }^{2}\right)$, must be non-negative. In contrast, $n^{2}>0$ is only an implicit assumption in paraxial theory [23].

\section{Mapping onto plane waves}

To derive the dark soliton of Eq. (2), one begins by writing $u$ in the form $u(\xi, \zeta)=\rho^{1 / 2}(\xi) \exp [i \Phi(\xi, \zeta)]$, where $\rho(\xi)$ and $\Phi(\xi, \zeta)$ are real functions. At this stage, $u$ is taken to comprise an on-axis grey dip with transverse intensity profile $\rho(\xi)$; the phase distribution $\Phi(\xi, \zeta)$ contains both dip and (off-axis) plane wave components. After substituting $u$ into Eq. (2) and collecting the real and imaginary parts, two quadrature equations are obtained:

$$
\begin{gathered}
\frac{2}{\rho} \frac{d^{2} \rho}{d \xi^{2}}-\frac{1}{\rho^{2}}\left(\frac{d \rho}{d \xi}\right)^{2}=8\left[\rho(1-\alpha \rho)+\frac{1}{2}\left(\frac{\partial \Phi}{\partial \xi}\right)^{2}\right. \\
\left.+\kappa\left(\frac{\partial \Phi}{\partial \zeta}\right)^{2}+\frac{\partial \Phi}{\partial \zeta}\right] \\
\kappa \rho \frac{\partial^{2} \Phi}{\partial \zeta^{2}}+\frac{\partial}{\partial \xi}\left(\rho \frac{\partial \Phi}{\partial \xi}\right)=0 .
\end{gathered}
$$


Since the dip is on-axis, $\Phi$ is at most a linear function of $\zeta$ (due to the plane wave component with velocity $V_{0}$ ) and the first term in Eq. (6b) vanishes to leave the familiar result $(\partial / \partial \xi)(\rho \partial \Phi / \partial \xi)=0$. This simplified equation yields

$$
\rho \frac{\partial \Phi}{\partial \xi}=C_{1}
$$

where $C_{1}$ is a (real) constant since the left-hand side is a function only of $\xi$. The subsequent integration of the Eqs. (6a) and (6c) is performed by following a similar procedure to that in Ref. [23]. The major modification with our calculation comes from recognizing the components of the Helmholtz plane wave background [solution (5)], and building these components explicitly into the structure of the desired solution $u$ via the dark-soliton boundary conditions.

The boundary conditions on $\rho$ are taken to be $\rho=\rho_{m}$ and $d \rho / d \xi=0$ at $\xi=0$ (beam centre); $\rho=\rho_{0}$ and $d \rho / d \xi=0$ as $|\xi| \rightarrow \infty$ (beam extremes), where $\rho_{0}>\rho_{m}$ is the plane-wave intensity. Applying these conditions to the derivative $\partial \Phi / \partial \xi$, one can identify

$$
\lim _{|\xi| \rightarrow \infty} \frac{\partial \Phi}{\partial \xi}=\frac{C_{1}}{\rho_{0}} \equiv \mp V_{0} \sqrt{\frac{1-4 \kappa \rho_{0}\left(1-\alpha \rho_{0}\right)}{1+2 \kappa V_{0}^{2}}},
$$

This result follows from the fact that the transverse slope of the dip's phase profile is zero at spatial infinity, so that only the plane-wave contribution survives [c.f., Eq. (3b)]. In this way, the dark soliton is mapped directly onto a plane wave. A second integration yields the formal solution

$$
\Phi(\xi, \zeta)=C_{1} \int_{-\infty}^{\xi} \frac{d q}{\rho(q)}+k_{\zeta} \zeta
$$

From solution (5), one can also identify 


$$
k_{\zeta} \equiv \frac{1}{2 \kappa}\left[-1 \pm \sqrt{\frac{1-4 \kappa \rho_{0}\left(1-\alpha \rho_{0}\right)}{1+2 \kappa V_{0}^{2}}}\right]
$$

where, in combination with Eq. (7), the upper (lower) signs describe a Helmholtz plane wave that is propagating in the forward (backward) direction (see Fig. 1). By introducing $\kappa k_{\zeta}^{2}+k_{\zeta} \equiv \beta$, Eq. (6a) reduces to

$$
\frac{d}{d \rho}\left[\frac{1}{\rho}\left(\frac{d \rho}{d \xi}\right)^{2}\right]=8\left[\rho(1-\alpha \rho)+\frac{1}{2}\left(\frac{\partial \Phi}{\partial \xi}\right)^{2}+\beta\right],
$$

which is formally identical to the intensity quadrature equation in Ref. [23]. Exact expressions for $\rho$ and $\Phi$ can now be found by integrating Eqs. (10) and (8), respectively. Two key algebraic results that help complete the Helmholtz solution are $C_{1}=\rho_{0}^{3 / 2}\left\{\left(1-a^{2}\right)\left[1-2 \alpha \rho_{0}\left(1-a^{2} / 3\right)\right]\right\}^{1 / 2}$ and $\beta=-1 / 2 \rho_{0}\left(3-a^{2}\right)+\alpha \rho_{0}^{2}\left(6-4 a^{2}+a^{4}\right) / 3$, where $a^{2} \equiv 1-\rho_{m} / \rho_{0}$. The plane-wave dispersion relation also provides a useful check on self-consistency, confirming that $-1 / 2 k_{\xi}^{2}-\rho_{0}\left(1-\alpha \rho_{0}\right)=\kappa k_{\zeta}^{2}+k_{\zeta}$ is indeed equal to $\beta$.

\section{Helmholtz dark solitons}

Before presenting the more general (off-axis) dark soliton solution (which is obtained from rotational transformations $[26,32]$ ) we first consider beam geometry in the laboratory frame - a schematic diagram is shown in Fig. 2. The plane wave propagates at angle $\theta=\tan ^{-1}\left[(2 \kappa)^{1 / 2} V\right]$ relative to the reference direction, while the grey dip propagates at angle $\theta_{0}=\tan ^{-1}\left[(2 \kappa)^{1 / 2} V_{0}\right]$ relative to this background. The propagation angle of the dip with respect to the reference direction is thus $\theta-\theta_{0}=$ $\tan ^{-1}\left[(2 \kappa)^{1 / 2} W\right]$. Here, $V, V_{0}$ and $W$ are the transverse, intrinsic, and net velocities, respectively. The dark soliton of Eq. (2) is 


$$
\begin{aligned}
u(\xi, \zeta) & =\rho^{1 / 2}(\xi, \zeta) \exp \left[\frac{i}{2} \sin ^{-1}\left(\frac{A(\xi, \zeta)-b}{C(\xi, \zeta)}\right)\right] \\
& \times \exp \left[ \pm i \sqrt{\frac{1-4 \kappa \rho_{0}\left(1-\alpha \rho_{0}\right)}{1+2 \kappa V^{2}}}\left(-V \xi+\frac{\zeta}{2 \kappa}\right)\right] \\
& \times \exp \left(-i \frac{\zeta}{2 \kappa}\right)
\end{aligned}
$$

where

$$
\begin{gathered}
\rho(\xi, \zeta)=\rho_{0}-\frac{2 d}{1-\frac{8}{3} \alpha \rho_{0}+\left[1-\frac{4}{3} \alpha \rho_{0}\left(2-a^{2}\right)\right] \cosh \Theta}, \\
\Theta(\xi, \zeta)=2 d^{1 / 2} \frac{\xi+W \zeta}{\sqrt{1+2 \kappa W^{2}}}, \\
A(\xi, \zeta)=\left(1-\frac{4}{3} \alpha \rho_{0}\right) \rho(\xi, \zeta), \\
b=2 \rho_{0}\left(1-a^{2}\right)\left[1-\frac{2}{3} \alpha \rho_{0}\left(3-a^{2}\right)\right], \\
C(\xi, \zeta)=\left[1-\frac{4}{3} \alpha \rho_{0}\left(2-a^{2}\right)\right] \rho(\xi, \zeta), \\
d=\rho_{0} a^{2}\left[1-\frac{2}{3} \alpha \rho_{0}\left(4-a^{2}\right)\right], \\
V_{0}^{2}=\frac{V-V_{0}}{1-2 \kappa \rho_{0}\left[3-a^{2}-\frac{2}{3} \alpha \rho_{0}\left(6-4 a^{2}+a^{4}\right)\right]},
\end{gathered}
$$

where $a^{2}$ is a contrast parameter $(0 \leq a \leq 1)$ and $W$ is the net velocity. The upper (lower) sign in Eq. (11a) corresponds to a forward (backward) solution (see Fig. 2).

The intensity profile of the $\operatorname{dip} \rho(\xi, \zeta)$ modulates the soliton phase through both $A(\xi, \zeta)$ and $C(\xi, \zeta)$, which are different for $a \neq 1$. The parameter $b$ is a constant determined solely by the plane-wave asymptotics, but which influences the size of the grey soliton intrinsic velocity. Towards the small-amplitude grey soliton limit $(a=0)$, 
the inverse-width factor $d$ tends to zero and the dip becomes very broad. For $\alpha>0$, black solitons $(a=1)$ can also be significantly delocalized when $\rho_{0}$ increases toward the modulational instability threshold of $1 / 2 \alpha$ (they cease to exist at, and above, this threshold). Also for $a>0$, where such plane-wave instability can occur, grey solitons are predicted to exist only below the instability threshold (see Section III for further details). We note that the Helmholtz Kerr dark soliton [32] is obtained from solution (11) when $\alpha=0$.

Potentially-dominant Helmholtz corrections arise from the finite propagation angles $\theta$ and $\theta_{0}$ [20,26,31,32]. These corrections can be of any order, even though $\kappa \sim$ $\varepsilon^{2}<<O(1)$. For instance, when $\left|\theta-\theta_{0}\right|=60^{\circ}$, an observer in the $(x, z)$ frame perceives the beam width $\Lambda_{\theta}=\left[1+\tan ^{2}\left(\theta-\theta_{0}\right)\right]^{1 / 2} \Lambda_{0}$ to have doubled relative to its on-axis value $\Lambda_{0} \equiv 1 / 2 d^{1 / 2}$ (see Fig. 3). In the limiting case of $\left|\theta-\theta_{0}\right| \rightarrow 90^{\circ}$, the grey soliton appears to be infinitely wide in $x$ since the dip is propagating perpendicularly to the reference axis. Angular corrections are not just of geometrical significance. They are central to optical contexts such as nonlinear beam interactions [4] and soliton refraction [5], where broadening can lead to corrections to paraxial predictions exceeding $100 \%$, and even give rise to new regimes of behaviour.

Dark soliton (11) can be represented in a more symmetric form by exploiting the relationships between velocities and propagation angles. By eliminating $V, V_{0}$ and $W$, one can combine both the forward and backward solutions into a single beam: 


$$
\begin{aligned}
u(\xi, \zeta) & =\rho^{1 / 2}(\xi, \zeta) \exp \left[\frac{i}{2} \sin ^{-1}\left(\frac{A(\xi, \zeta)-b}{C(\xi, \zeta)}\right)\right] \\
& \times \exp \left[i \sqrt{\frac{1-4 \kappa \rho_{0}\left(1-\alpha \rho_{0}\right)}{2 \kappa}}\right. \\
& \left.\times\left(-\xi \sin \theta+\frac{\zeta}{\sqrt{2 \kappa}} \cos \theta\right)\right] \\
& \times \exp \left(-i \frac{\zeta}{2 \kappa}\right), \\
\Theta(\xi, \zeta)= & 2 d^{1 / 2}\left[\xi \cos \left(\theta-\theta_{0}\right)+\frac{\zeta}{\sqrt{2 \kappa}} \sin \left(\theta-\theta_{0}\right)\right] .
\end{aligned}
$$

Here, the propagation angle of the plane wave is now bounded by $-180^{\circ} \leq \theta \leq+180^{\circ}$, but the intrinsic propagation angle (defined with respect to the plane wave background) still satisfies $-90^{\circ} \leq \theta_{0} \leq+90^{\circ}$.

\section{E. Bistable characteristics}

By looking at the solution continuum in Eqs. (11a)-(11i), one can identify pairs of beams where the grey dips have the same FWHM, but the plane-wave backgrounds have different intensities [23]. Such non-degenerate pairs can be obtained from the condition $\rho(s=v \Delta)=\left(\rho_{0}+\rho_{m}\right) / 2$, where $s \equiv(\xi+W \zeta) /(1+$ $\left.2 \kappa W^{2}\right)^{1 / 2}, \Delta=\operatorname{sech}^{-1}\left(2^{-1 / 2}\right) \approx 0.8814$, and $v$ measures the half-width in units of $\Delta$ $[20,23]:$

$$
\begin{aligned}
\rho_{0}^{1 / 2} & =\frac{1}{2 v \Delta a\left[1-\frac{2}{3} \alpha \rho_{0}\left(4-a^{2}\right)\right]^{1 / 2}} \\
& \times \cosh ^{-1}\left[\frac{3-\frac{8}{3} \alpha \rho_{0}\left(3-a^{2}\right)}{1-\frac{4}{3} \alpha \rho_{0}\left(2-a^{2}\right)}\right] .
\end{aligned}
$$

Canonical solutions are defined by $v=1$, where the half-width-at-half-maximum is $s$

$=\Delta$, and the FWHM is thus $2 \Delta$. There is no $\kappa$ or $\kappa V^{2}$ dependence in Eq. (13). This 
follows from the fact that the FWHM of a beam is defined in a direction perpendicular to the propagation direction; bistability is essentially an intrinsic (direction-independent) property of the solitons. Thus, plane-wave intensities must be insensitive to the relative orientation of the observer's coordinate axes.

Figure 4 shows that when $\alpha$ is less than some maximum value, there are two values of $\rho_{0}$ that satisfy Eq. (13) for a given contrast parameter $a$. As $\alpha \rightarrow 0$, the lower solution branch tends toward $\rho_{0}{ }^{1 / 2} \sim 1 / v a$, while the upper branch has a cut-off at coordinates $\left(\alpha_{\text {crit }}, \rho_{0 \text { crit }}{ }^{1 / 2}\right)$, where $\alpha_{\text {crit }}=\left(3 v^{2} \Delta^{2} / 2\right) a^{4}\left(4-a^{2}\right)^{-2}$ and $\rho_{0 \text { crit }}=(4-$ $\left.a^{2}\right) / v^{2} \Delta^{2} a^{4}$ (an approximate cut-off point was given in Ref. [23]).

\section{F. Recovery of paraxial solitons}

Herrmann's paraxial soliton [23] can be recovered from the forward Helmholtz solution (11) after careful consideration of a quite subtle multiple limit, specifically $\kappa \rightarrow 0$ (broad beams), $\kappa \rho_{0} \rightarrow 0$ (moderate intensities), $\kappa V^{2} \rightarrow 0$ and $\kappa V_{0}^{2} \rightarrow 0$ (near-negligible propagation angles). One finds that [33]

$$
\begin{gathered}
u(\xi, \zeta) \sim \rho^{1 / 2}(\xi, \zeta) \exp \left[\frac{i}{2} \sin ^{-1}\left(\frac{A(\xi, \zeta)-b}{C(\xi, \zeta)}\right)\right] \\
\times \exp \left\{-i V \xi-i\left[\rho_{0}\left(1-\alpha \rho_{0}\right)+\frac{V^{2}}{2}\right] \zeta\right\}, \\
\Theta(\xi, \zeta) \sim 2 d^{1 / 2}(\xi+W \zeta), \\
W \sim V-V_{0} \\
V_{0}^{2} \sim \rho_{0}\left(1-a^{2}\right)\left[1-\frac{2}{3} \alpha \rho_{0}\left(3-a^{2}\right)\right] .
\end{gathered}
$$

Note that when $\alpha=0$, the paraxial Kerr grey soliton is recovered from Eqs. (14a)(14d), where $V_{0}^{2}=\rho_{0}\left(1-a^{2}\right)$. By applying the same asymptotic procedure to the 
backward Helmholtz solution, one finds that a phase factor exp[-i2( $\zeta / 2 \kappa)]$ survives the limit process. This result emphasises the uni-directionality of paraxial theory, which has no analogue of backward waves.

Together, the four physical limits mentioned above define the paraxial approximation. That is, conventional modelling is valid when all contributions arising from the operator $\kappa \partial_{\zeta \zeta}$ are negligible simultaneously. If even one of the four criteria is relaxed, a Helmholtz description of nonlinear beams is necessary. We stress that one cannot obtain solution (14) from solution (11), nor the corresponding paraxial governing equation from Eq. (2), simply by letting $\kappa \rightarrow 0$.

\section{DARK SOLITON STABILITY}

The stability of plane-wave solutions to generic nonlinear Helmholtz equations has been analysed elsewhere [31]. Here, we consider the more interesting issue of soliton stability. In particular, we focus on the case $\alpha>0$, where bistability exists. Computer simulations [34] are used to analyse the fully nonlinear problem of soliton robustness against perturbations to the local beam shape.

\section{A. Black solitons}

We first consider launching an obliquely-propagating black beam $\left(a=1, V_{0}=0\right)$ of the form

$$
\begin{aligned}
u(\xi, 0) & =\left[\rho_{0}-\frac{2 d}{1-\frac{8}{3} \alpha \rho_{0}+\left(1-\frac{4}{3} \alpha \rho_{0}\right) \cosh (2 \sqrt{d} \xi)}\right]^{1 / 2} \\
& \times \exp \left(-i V \sqrt{\frac{1-4 \kappa \rho_{0}\left(1-\alpha \rho_{0}\right)}{1+2 \kappa V^{2}}} \xi\right),
\end{aligned}
$$


where the broadening factor $\left(1+2 \kappa V^{2}\right)^{1 / 2}=\sec \theta$ has been omitted from the intensity profile. This class of initial-value problem thus addresses what happens when an exact paraxial solution (14) with transverse velocity $V\left\{\left[1-4 \kappa \rho_{0}\left(1-\alpha \rho_{0}\right)\right] /\left(1+2 \kappa V^{2}\right)\right\}^{1 / 2}$ is launched into an inherently off-axis nonparaxial regime $[32,35]$.

For $\kappa=10^{-3}$ and $\kappa=10^{-4}$, we consider propagation angles of $|\theta|=15^{\circ},|\theta|=$ $30^{\circ}$ and $|\theta|=45^{\circ}$. For $\alpha=0.15$, the lower and upper solution branches have $\rho_{0} \approx$ 1.486 and $\rho_{0} \approx 3.018$, respectively (see Fig. 4). Figure 5 shows that as $\zeta \rightarrow \infty$, the perturbed beam transforms smoothly into a stationary Helmholtz beam with width $\Lambda_{\theta}$ $\sim\left(1+\tan ^{2} \theta\right)^{1 / 2} \Lambda_{0}$. Solutions lying on both branches can be interpreted as stable robust attractors $[20,31]$.

\section{B. Grey solitons}

Since dark soliton (11) should have a real width (dictating that $d>0$ ), the plane wave intensity $\rho_{0}$ is bounded by the inequality

$$
\rho_{0}<\frac{3}{2 \alpha\left(4-a^{2}\right)}
$$

This is an interesting result. For black solitons, when $a^{2}=1$, the maximum allowed $\rho_{0}$ falls exactly below the threshold for modulational instability. In the grey limit, when $a^{2}=0$, one has that $\rho_{0}<3 / 8 \alpha$, and the maximum $\rho_{0}$ in this case is well below the threshold value. When inequality (16) is met, then a second existence requirement (obtained from the condition $V_{0}^{2} \geq 0$ ), namely $\rho_{0}<3 /\left[2 \alpha\left(3-a^{2}\right)\right]$, is automatically satisfied.

The condition $d>0$ has a profound impact on the angular characteristics of the beam. By using Eq. (16) in conjunction with Eq. (11i), it has been found that even a 
very small value of $\alpha$ can place strong restrictions on the maximum allowable $\left|V_{0}\right|$. In turn, this limits the maximum supportable intrinsic propagation angle $\left|\theta_{0}\right|_{\max }$ (see Fig. $6)$; as $\kappa$ is reduced, $\left|\theta_{0}\right|_{\max }$ is depressed even further. Of course, the net propagation angle $\theta-\theta_{0}$ can still be highly nonparaxial. This new result provides further evidence of quantitative differences between the predictions of Helmholtz (finite- $\kappa$ ) and paraxial models.

Such restrictions on $\left|\theta_{0}\right|_{\max }$ are lifted when $\alpha<0$, and the nonlinearity is of a purely defocusing type [32]. For instance, one can attain $\left|\theta_{0}\right| \rightarrow 90^{\circ}$ (i.e., $V_{0}^{2} \rightarrow \infty$ ) by ramping-up the plane wave intensity towards its limiting value. From Eq. (11i), this value is found to be

$$
\rho_{0}=\frac{3-a^{2}}{\frac{4}{3}|\alpha|\left(6-4 a^{2}+a^{4}\right)}\left[\sqrt{1+\left(\frac{4|\alpha|}{3 \kappa}\right) \frac{6-4 a^{2}+a^{4}}{9-6 a^{2}+a^{4}}}-1\right] .
$$

Again, this type of large-angle regime has no counterpart in paraxial theory [23].

To address the stability properties of grey solitons, we consider the solution continuum without necessarily applying the half-width condition described in Section IIE. In this way, one can gain insight into the robustness of grey beams independently of any externally-imposed constraints. Another advantage with this approach is that the more general solution allows access to larger intrinsic propagation angles than would otherwise be supportable in the bistable solution (see Fig. 6).

Results are now presented beam for self-reshaping grey solitons with $\alpha=0.01$, $a=0.4$, and $\kappa=10^{-3}$ (so that $\left|\theta_{0}\right|_{\max } \approx 9^{\circ}$ and $\rho_{0}<39$ ). With these parameters, plane wave intensities $\rho_{0}=4,10$, and 20 correspond to intrinsic propagation angles $\left|\theta_{0}\right| \approx$ $4.56^{\circ}, 6.83^{\circ}$, and $8.59^{\circ}$, respectively [profiles are shown in Fig. 7(a)]. The initial condition $u(\xi, 0)$ is obtained from solution (11) with $\Theta(\xi, 0)=2 d^{1 / 2} \xi$. This corresponds 
to an input beam whose width has been reduced by the Helmholtz factor $\left(1+2 \kappa V_{0}^{2}\right)^{1 / 2}$ $=\sec \theta_{0}$. The perturbed beam evolves into a stationary Helmholtz dark soliton, with the dip broadening toward the asymptotic value $\Lambda_{\infty} \sim\left(1+\tan ^{2} \theta_{0}\right)^{1 / 2} \Lambda_{0}$ [see Fig. 7(b)].

\section{CONCLUSIONS}

In this paper, a Helmholtz model has been proposed for describing broad (scalar) optical beams in a medium with nonlinear refractive index $n_{N L}\left(|E|^{2}\right)=-n_{2}|E|^{2}$ $+n_{4}|E|^{4}$. Two families of exact analytical dark soliton (corresponding to forward and backward beams) have been derived, and self-consistency of these solutions has been demonstrated. Their geometry and bistability characteristics have also been explored in full detail, and the predictions of paraxial theory recovered in a simultaneous fourfold limit. We have further found that these solitons exist only when the background plane wave is modulationally stable. Computer simulations have verified that cubicquintic Helmholtz dark solitons with $\alpha>0$ can be interpreted as robust attractors [31,32] (we note, in passing, that reshaping solitons with $\alpha<0$ exhibit the same type of qualitative behaviour as shown in Figs. 5 and 7).

The dark solutions studied in this paper complement their bright counterparts [20], and extend our earlier analyses [32] to more general classes of nonlinear materials. Solitons, and their wave equations, are universal features across many areas of nonlinear science. Specifically, we highlight that our results may play a key role in many future applications. For example, configurations involving bright spatial-soliton switching [36], logic [37], and dragging [38] could be extended to involve dark solitons. Solution (11) also opens up the possibility of additional novel studies involving angular geometries, such as interactions between bistable dark solitons, and also between bistable bright and dark solitons. Further considerations 
could link up with the field of soliton computing [39]. We also propose that bistability, in parallel with the recent analysis of arbitrary-angle beams-at-interfaces configurations [5], could give rise to qualitatively-new phenomena. For instance, even the behaviour of monostable dark solitons at interfaces appears to have received very little attention in the literature [40].

Modelling with Helmholtz equations [25,26] equips one with the necessary mathematical and computational tools for understanding oblique-propagation effects. It paves the way for designing a host of novel spatial soliton device architectures whose operational emphasis is on angular geometries. 


\section{REFERENCES}

[1] G. I. Stegeman and M. Segev, Science 286, 1518 (1999).

[2] V. E. Zakharov and A. B. Shabat, Sov. Phys. JETP 34, 62 (1972); ibid. 37, 823 (1973); J. P. Gordon, Opt. Lett. 8, 596 (1983); M. J. Ablowitz et al., J. Opt. Soc. Am. B 14, 1788 (1997); O. Cohen et al., Opt. Lett. 27, 2013 (2002); Phys. Rev. Lett. 89, 133901 (2002).

[3] A. B. Aceves, J. V. Moloney, and A. C. Newell, Phys. Rev. A 39, 1809 (1989); ibid. 39, 1828 (1989); Phys. Lett. A 129, 231 (1988); Opt. Lett. 13, 1002 (1988).

[4] P. Chamorro-Posada and G. S. McDonald, Phys. Rev. E 74, 036609 (2006).

[5] J. Sánchez-Curto, P. Chamorro-Posada, and G. S. McDonald, J. Opt. A: Pure Appl. Opt. 11, 054015 (2009); Opt. Lett. 32, 1126 (2007).

[6] P. Mandel, M. Georgiou, and T. Erneux, Phys. Rev. A 47, 4277 (1993); T. Erneux and P. Mandel, ibid. 28, 896 (1983).

[7] L. A. Lugiato and C. Oldano, Phys. Rev. A 37, 3896 (1988); R. Bonifacio and L. A. Lugiato, ibid. 18, 1129 (1978).

[8] G. S. McDonald and W. J. Firth, J. Opt. Soc. Am. B 10, 1081 (1993); ibid. 7, 1328 (1990); J. Mod. Opt. 37, 613 (1990).

[9] A. E. Kaplan, Sov. Phys. JETP 45, 896 (1977); JETP Lett. 24, 114 (1976);

[10] P. W. Smith and W. J. Tomlinson, IEEE J. Quantum Electron. QE-20, 30 (1984); P. W. Smith et al., Appl. Phys. Lett. 35, 846 (1979).

[11] A. E. Kaplan, Phys. Rev. Lett. 55, 1291 (1985); IEEE J. Quantum Electron. QE-21, 1538 (1985); A. E. Kaplan and C. T. Law, IEEE J. Quantum Electron. QE-21, 1529 (1985).

[12] J. A. Goldstone and E. Garmire, Phys. Rev. Lett. 53, 910 (1984). 
[13] T. T. Shi and S. Chi, Opt. Lett. 15, 1123 (1990).

[14] W. J. Firth et al., J. Opt. Soc. Am. B 19, 747 (2002).

[15] R. H. Enns, S. S. Rangnekar, and A. E. Kaplan, Phys. Rev. A 36, 1270 (1987); Phys. Rev. Lett. 57, 778 (1986); A. E. Kaplan, Phys. Rev. Lett. 57, 779 (1986).

[16] R. H. Enns, S. S. Rangnekar, and A. E. Kaplan, Phys. Rev. A 35, 466 (1987); R. H. Enns and S. S. Rangnekar, Opt. Lett. 12, 108 (1987); IEEE J. Quantum Electron. QE-23, 1199 (1987).

[17] S. Gatz and J. Herrmann, IEEE J. Quantum Electron. 28, 1732 (1992); J. Hermann, Opt. Commun. 87, 161 (1992).

[18] S. Gatz and J. Herrmann, Opt. Lett. 17, 484 (1992); J. Opt. Soc. Am. B 8, 2296 (1991); J. Herrmann, ibid. 8, 1507 (1991).

[19] W. Krolikowski and B. Luther-Davies, Opt. Lett. 17, 1414 (1992).

[20] J. M. Christian, G. S. McDonald, and P. Chamorro-Posada, Phys. Rev. A 76, 033833 (2007); J. Opt. Soc. Am. B 26, 2323 (2009).

[21] F. G. Bass, V. V. Konotop, and S. A. Puzenko, Phys. Rev. A 46, 4185 (1992);

L. J. Mulder and R. H. Enns, IEEE J. Quantum Electron. 25, 2205 (1989).

[22] W. Krolikowski and B. Luther-Davies, Opt. Lett. 18, 188 (1993).

[23] J. Herrmann, Opt. Commun. 91, 337 (1992).

[24] D. E. Pelinovsky, Y. S. Kivshar, and V. V. Afanasjev, Phys. Rev. E 54, 2015 (1996); Y. S. Kivshar and X. Yang, Phys. Rev. E 49, 1657 (1994).

[25] T. A. Laine and A. T. Friberg, J. Opt. Soc. Am. B 17, 751 (2000); A. P. Sheppard and M. Haelterman, Opt. Lett. 23, 1820 (1998); G. Fibich, Phys. Rev. Lett. 76, 4356 (1996); M. D. Feit and J. A. Fleck, J. Opt. Soc. Am. B 5, 633 (1988). 
[26] P. Chamorro-Posada, G. S. McDonald, and G. H. C. New, J. Opt. Soc. Am. B 19, 1216 (2002); J. Mod. Opt. 45, 1111 (1998).

[27] M. Lax, W. H. Louisell, and W. B. McKnight, Phys. Rev. A 11, 1365 (1975).

[28] S. Chi and Q. Guo, Opt. Lett. 20, 1598 (1995).

[29] A. Ciattoni et al., Opt. Lett. 30, 516 (2005); B. Crosignani, A. Yariv, and S. Mookherjea, ibid. 29, 1254 (2004); A. Ciattoni et al., J. Opt. Soc. Am. B 17, 809 (2000).

[30] B. A. Malomed et al., Phys. Rev. A 64, 023814 (2001); E. Granot et al., Opt. Commun. 178, 431 (2000); Opt. Lett. 22, 1290 (1997).

[31] J. M. Christian, G. S. McDonald, and P. Chamorro-Posada, J. Phys. A: Math. Theor. 40, 1545 (2007); 40, 8601 (2007)

[32] P. Chamorro-Posada and G. S. McDonald, Opt. Lett. 28, 825 (2003).

[33] In this paper, we have chosen the beam alignment used in Ref. [32]. A consequence is that solution (14) is mathematically different from Hermann's paraxial soliton, although it is physically equivalent.

[34] P. Chamorro-Posada, G. S. McDonald, and G. H. C. New, Opt. Commun. 192, $1(2001)$.

[35] P. Chamorro-Posada, G. S. McDonald, and G. H. C. New, J. Mod. Opt. 47, 1877 (2000).

[36] Y. W. Wu, Opt. Express. 14, 4005 (2006); Appl. Opt. 44, 4144 (2005); F. Garzia, C. Sibilia, and M. Bertolotti, J. Lightwave Technol. 18, 1036 (2001).

[37] S. Blair and K. Wagner, Appl. Opt. 39, 6006 (2000); 38, 6749 (1999).

[38] J. U. Kang, G. I. Stegeman, A. Villeneuve, and J. S. Aitchison, Pure Appl. Opt. 5583 (1996); J. U. Kang, G. I. Stegeman, and J. S. Aitchison, Opt. Lett. 21, 189 (1996); S. Blair and K. Wagner, ibid. 19, 194 (1994). 
[39] C. Anastassiou et al., Phys. Rev. Lett. 83, 2332 (1999); M. H. Jakubowski, K. Steiglitz, and R. Squier, Phys. Rev. E 58, 6752 (1998).

[40] J. Sánchez-Curto, P. Chamorro-Posada, and G. S. McDonald, "Helmholtz dark solitons at nonlinear interfaces," submitted. 


\section{FIGURE CAPTIONS}

FIG. 1. (Color online) Geometry of (a) forward and (b) backward Helmholtz plane waves [solution (5)] in the laboratory frame. In both cases, the velocity $V>0$ and the magnitude of the transverse projection of the (dimensionless) wavevector, $\left|k_{\xi}\right|$, is bounded by $\left|k_{\xi}\right| \leq\left|k_{\xi}\right|_{\max }$, where $\left|k_{\xi}\right|_{\max }=\left\{\left[1-4 \kappa \rho_{0}\left(1-\alpha \rho_{0}\right)\right] / 2 \kappa\right\}^{1 / 2}$. This limit corresponds to $|V| \rightarrow \infty$, or equivalently $|\theta| \rightarrow 90^{\circ}$, where the wave propagates along the $x$ axis (that is, perpendicularly to the reference direction).

FIG. 2. (Color online) Schematic diagram showing the geometry of the Helmholtz dark soliton in the laboratory frame. (a) A black solution ( $a=1$, thus $V_{0}=0$ and there is an absolute-zero in the field at the beam centre), and (b) a grey solution $(0<a<1$, $\left.\left|V_{0}\right|>0\right)$. The plane-wave background propagates at angle $\theta$ relative to the $z$ axis, while the propagation angle $\theta_{0}$ of the grey dip is specified relative to the background. The angle of the dip relative to the $z$ axis is thus $\theta-\theta_{0}$. Parts (c) and (d) illustrate the corresponding backward-propagating beam.

FIG. 3. (Color online) Angular beam broadening for black $\left(a=1, V_{0}=0\right)$ Helmholtz solitons with $\alpha=0.15$ on the (a) upper and (b) lower solution branches (see Section II.E), where $\rho_{0} \approx 3.018$ and 1.486 , respectively. The modulational instability threshold intensity for this value of $\alpha$ is $\rho_{0}=1 / 2 \alpha \approx 3.33$, so the plane-wave background of both solitons is modulationally stable. Solid line (black): $\theta=0^{\circ}$ (paraxial profile); dashed line (blue): $|\theta|=30^{\circ}$; dotted line (red): $|\theta|=45^{\circ}$, dot-dash line (green): $|\theta|=60^{\circ}$. 
FIG. 4. (Color online) Bistability characteristic for the Helmholtz soliton (11), as defined by the implicit equation (13). Solutions lying on the upper and lower branches have the same FWHM ( $v=1$, so $s=\Delta)$ but different plane-wave intensities.

FIG. 5. (Color online) Asymptotic transformation of the perturbed beam (15) into an exact (stationary) Helmholtz soliton for (a) lower- and (b) upper-branch solutions, where $\rho_{0} \approx 1.486$ and 3.018 , respectively. Solid line (black): $|\theta|=15^{\circ}$; dashed line (blue): $|\theta|=30^{\circ}$; dot-dash line (red): $|\theta|=45^{\circ}$. Horizontal bars denote theoretical predictions $\Lambda_{\theta} \sim\left(1+\tan ^{2} \theta\right)^{1 / 2} \Lambda_{0}$, where $\Lambda_{0}=1 / 2 d^{1 / 2}$

FIG. 6. (Color online) Maximum intrinsic propagation angle $\left|\theta_{0}\right|_{\max }$ as a function of the contrast parameter $a$ when $\alpha=0.01$ (black solid line), $\alpha=0.05$ (blue dashed line), $\alpha=0.10$ (red dotted line), and $\alpha=0.15$ (green dot-dash line). The nonparaxial parameter is $\kappa=10^{-3}$. When $\alpha=0$ (a defocusing Kerr nonlinearity), $\left|\theta_{0}\right|_{\max }=90^{\circ}$ for $0<a<1$.

FIG. 7. (Color online) (a) Exact Helmholtz grey soliton profiles when $\alpha=0.01, a=$ 0.4 , and $\kappa=10^{-3}$. The plane-wave intensities are $\rho_{0}=4$ (black solid line), $\rho_{0}=10$ (blue dashed line), and $\rho_{0}=20$ (red dot-dashed line), which correspond to $\left|\theta_{0}\right|$ $\approx 4.56^{\circ}, 6.83^{\circ}$, and $8.59^{\circ}$, respectively. (b) Transformation of the perturbed input beam [where the broadening factor $\left(1+2 \kappa V_{0}^{2}\right)^{1 / 2}$ is omitted from the profile] into an exact (stationary) Helmholtz soliton. The horizontal bars denote asymptotic predictions for the relative beam width $\Lambda_{\infty} / \Lambda_{0} \sim\left(1+\tan ^{2} \theta_{0}\right)^{1 / 2}$ as $\zeta \rightarrow \infty$ (where $\Lambda_{0}=$ $\left.1 / 2 d^{1 / 2}\right)$. The numerical data has been fitted to an adiabatic trial solution. This 
approximation method is generally valid for perturbed black solitons (see Fig. 5), and also for grey solitons as $\zeta \rightarrow \infty$. However, it can become slightly less reliable for modelling the initial stages of grey-beam evolution (denoted by the grey band) due to the emission of radiation. 
FIGURE 1

J. M. Christian et al.

Submitted to Physical Review A

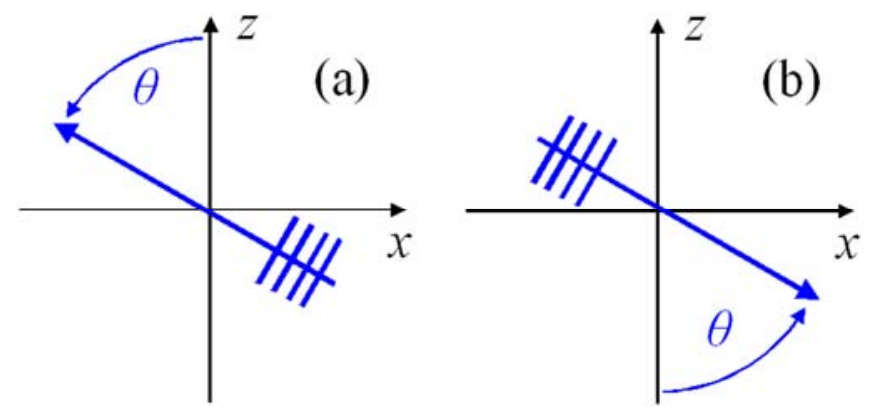


FIGURE 2

J. M. Christian et al.

Submitted to Physical Review A
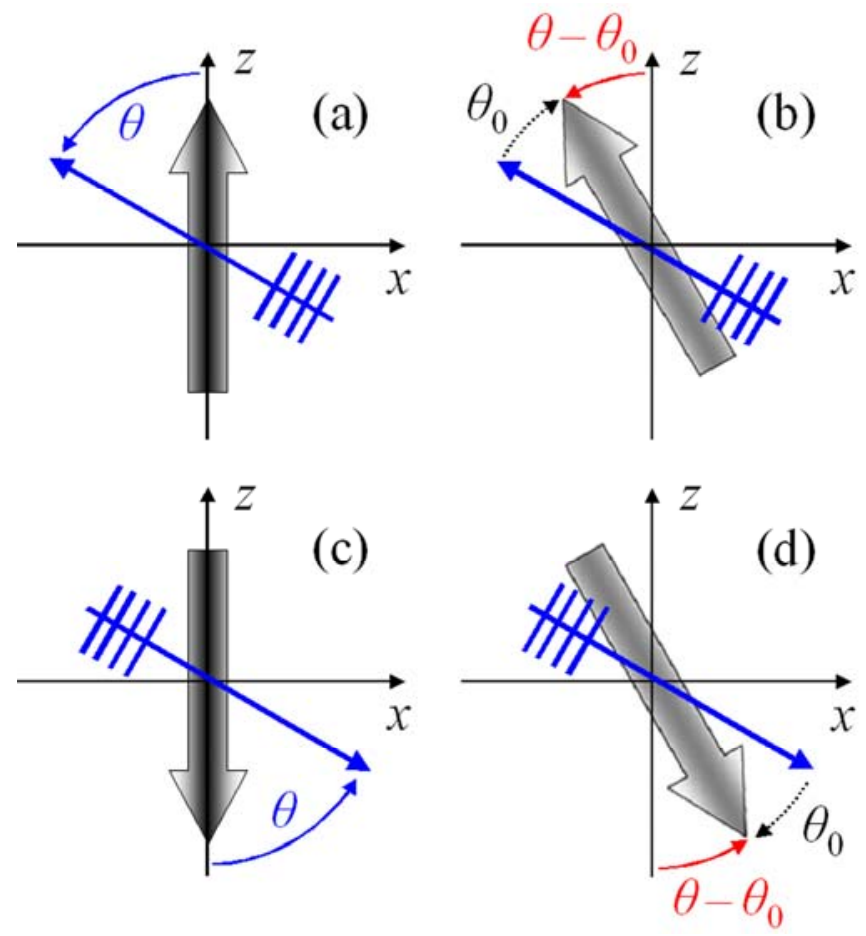
FIGURE 3

J. M. Christian et al.

Submitted to Physical Review A
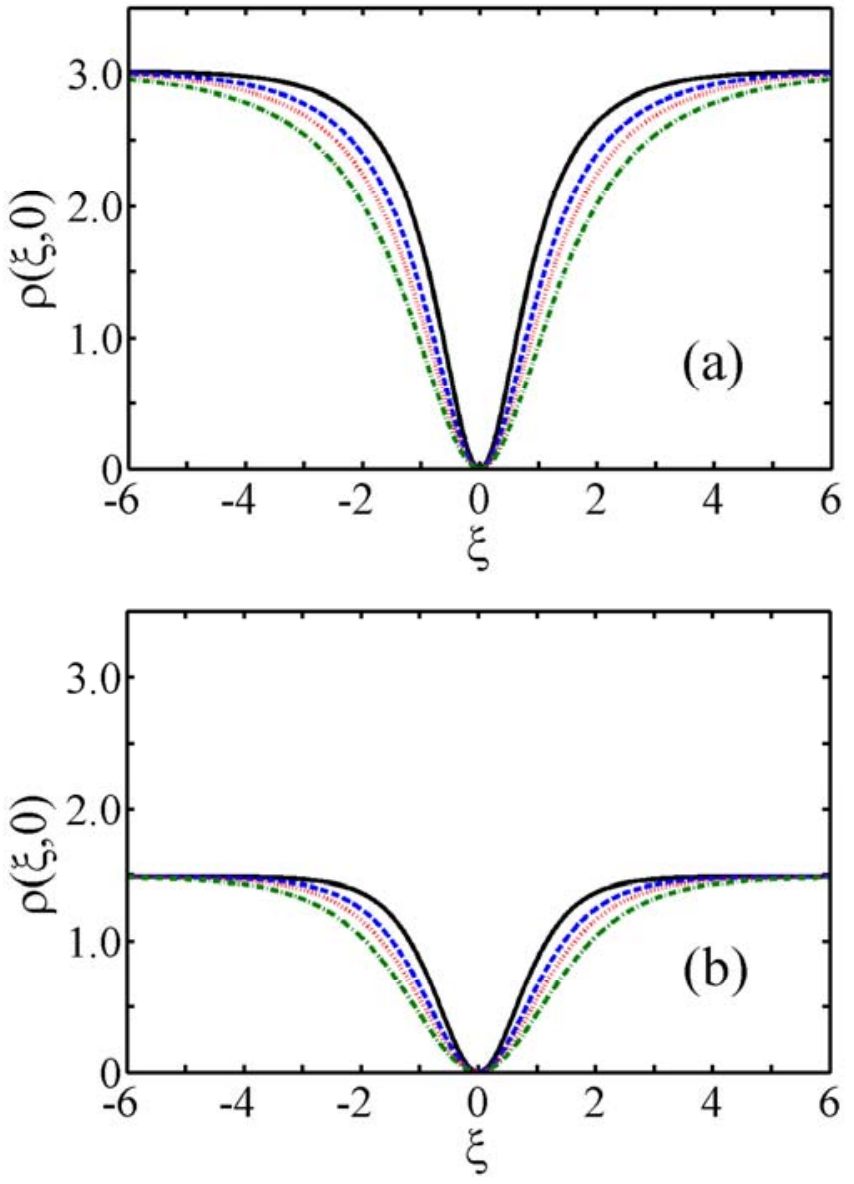
FIGURE 4

J. M. Christian et al.

Submitted to Physical Review A

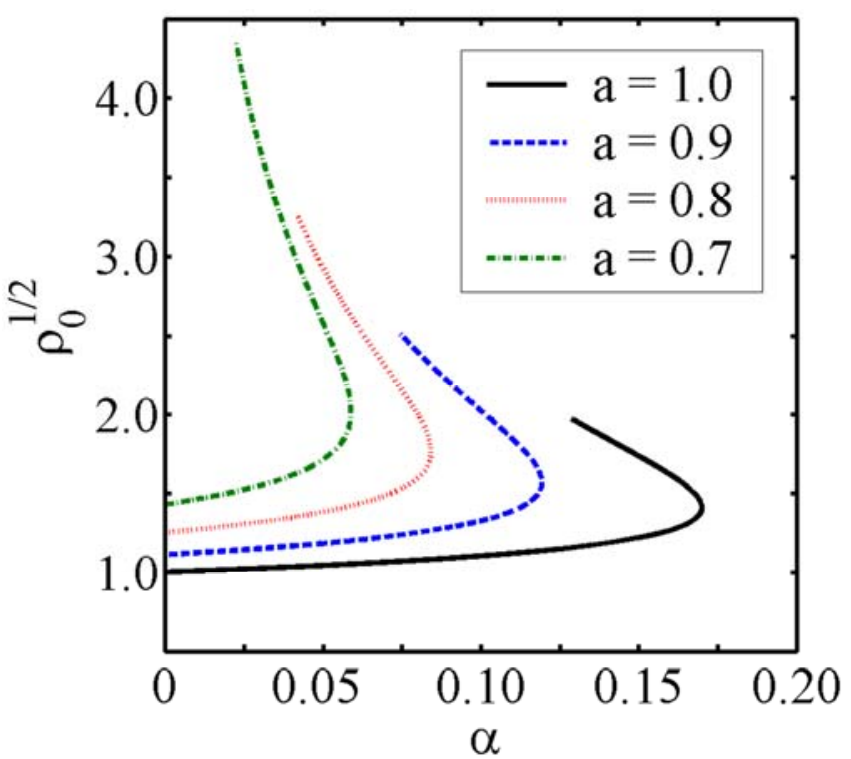


FIGURE 5

J. M. Christian et al.

Submitted to Physical Review A
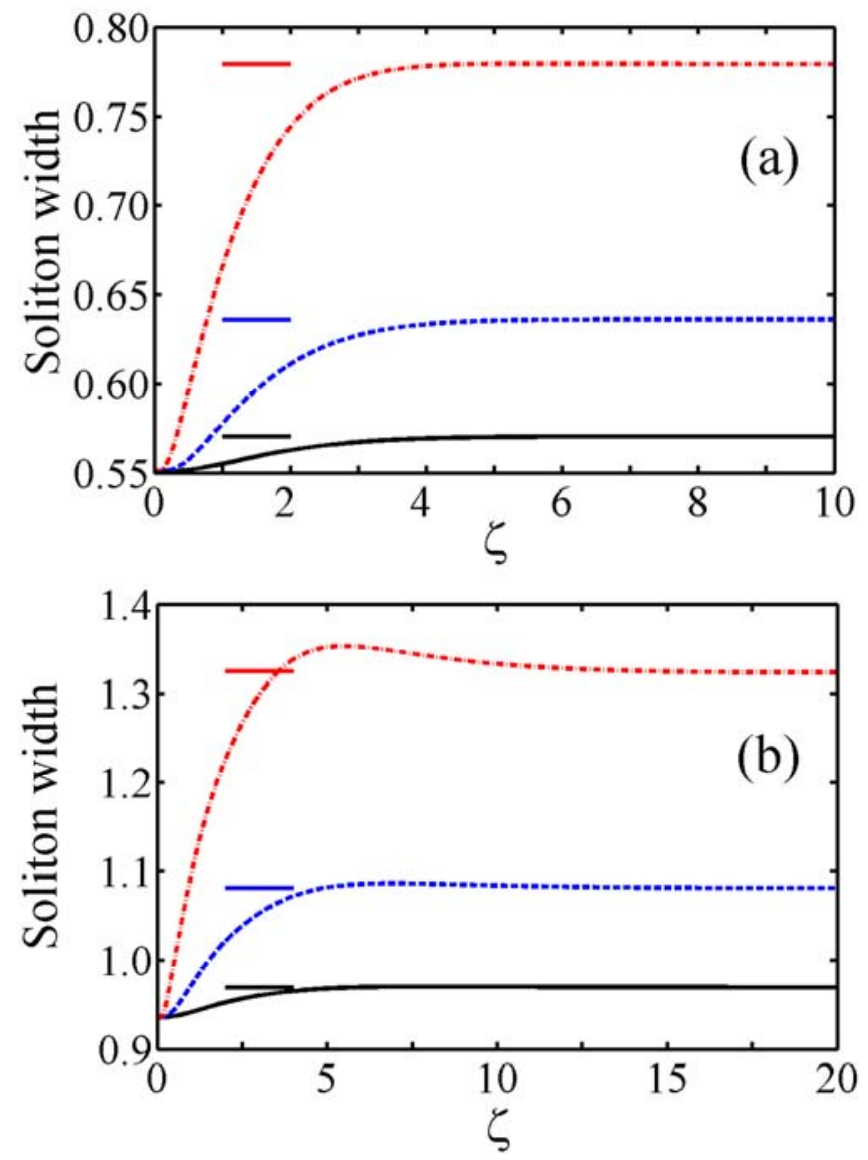
FIGURE 6

J. M. Christian et al.

Submitted to Physical Review A

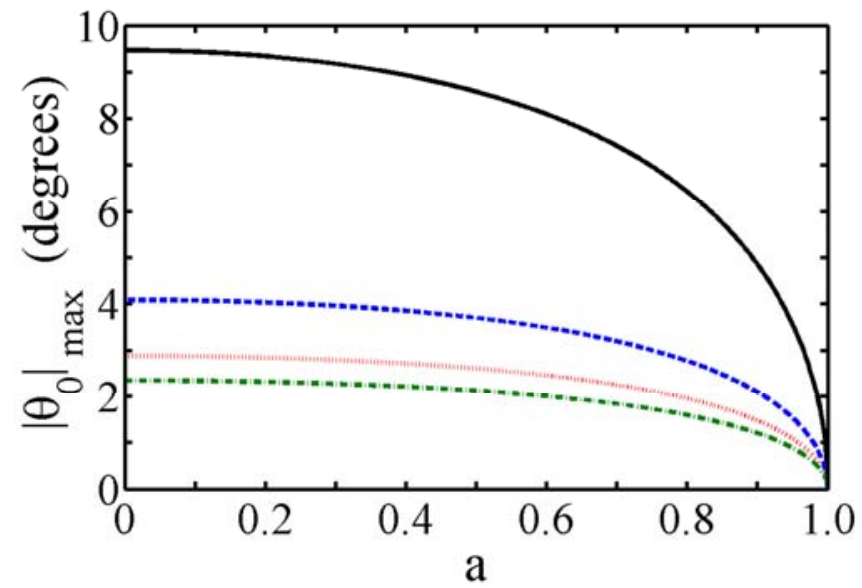


FIGURE 7

J. M. Christian et al.

Submitted to Physical Review A
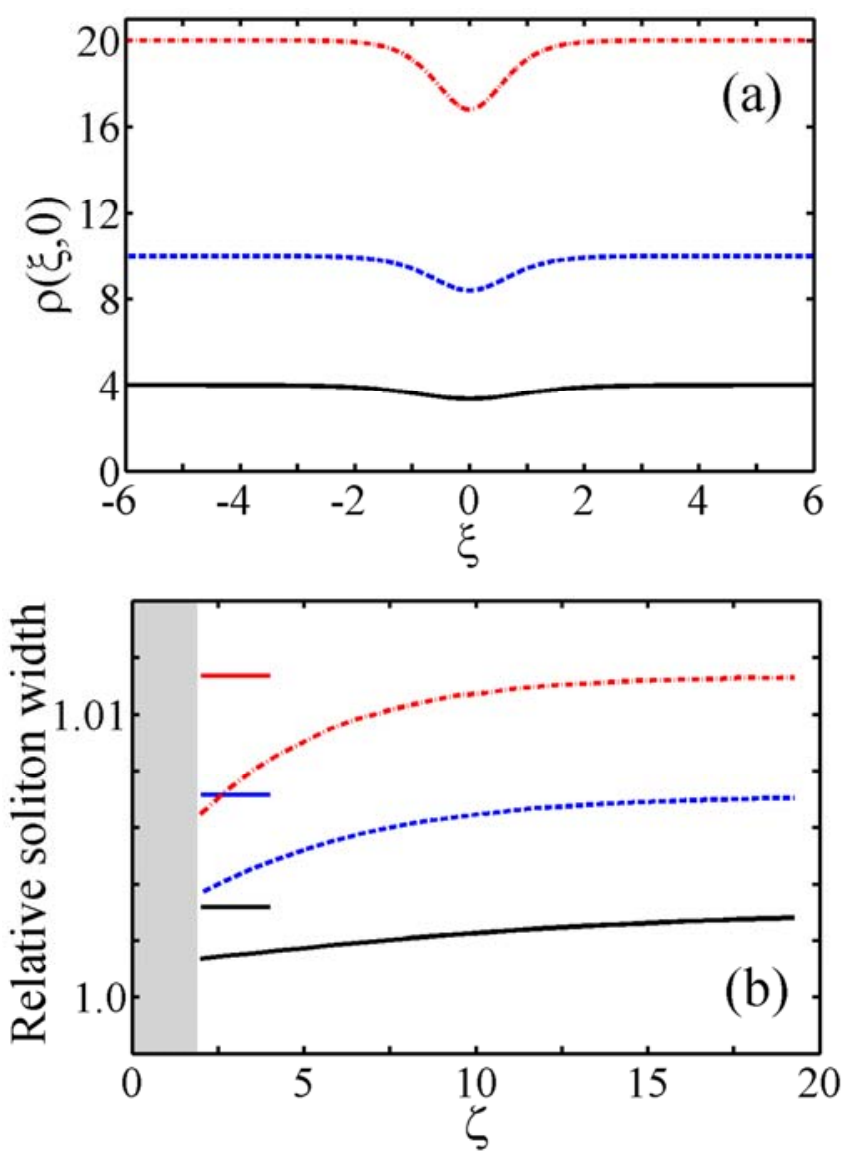